\title{
Evolución de los enfoques teóricos de la innovación desde la perspectiva de Schumpeter
}

\section{Innovation's theoretical Schumpeter's perspective

\author{
approaches evolution from
}

Ulises Mendoza Arvizo*, Andrés Hernández Gómez** y Lázaro Rico Pérez***

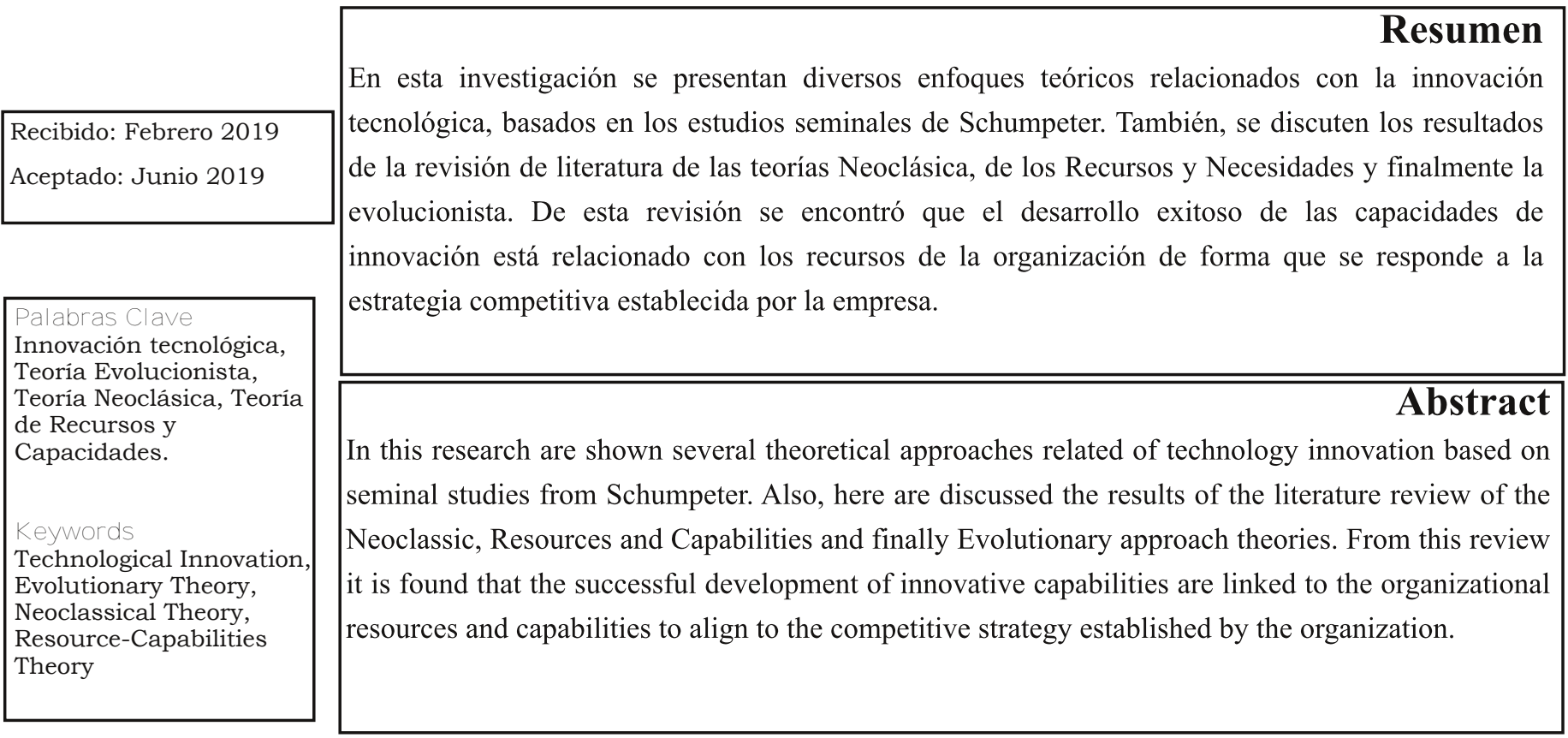

\section{Introducción}

Es Schumpeter (1934), el autor pionero y propulsor de la escuela evolucionista en el tema de la innovación, desde sus primeras afirmaciones acerca de que el tamaño de la empresa era un factor significativo en el desarrollo de innovaciones. Diferentes autores, como se muestran durante el texto, se han basado en él para sus investigaciones relacionadas con el tema de estudio.

La escuela evolucionista propone empresas diferenciadas tecnológicamente, con habilidades distintas para desarrollar cosas, con diversas capacidades de aprendizaje y procesamiento de la información. A diferencia de la neoclásica que considera a la innovación como un factor estático, es decir, sin variabilidad, fácil de reproducirse.

* Doctor en Ciencias de la Administración por la Universidad Nacional Autónoma de México, Catedrático en la Universidad Autónoma de Ciudad Juárez

** Doctor en Ciencias de la Administración por la Universidad Nacional Autónoma de México, Catedrático en la Universidad Autónoma de Ciudad Juárez

*** Doctor en Ciencias de la Ingeniería Industrial por el Instituto Tecnológico de Ciudad Juárez Catedrático en la Universidad Autónoma de Ciudad Juárez 


\section{Teorías que sustentan la innovación tecnológica}

La mayor parte de los estudios realizados sobre innovación tecnológica tiene su fundamento en la experiencia de sus autores y no en una teoría específica. Sin embargo, hacen referencia a los autores más reconocidos en el tema, como: Daft (1978), Damanpour (1991), Dosi (1997), Sohal et al. (1999), Teece (1986) y quienes a su vez basan sus afirmaciones en Schumpeter (1934). Esta referencia de autores permite asumir a éste último como el autor base de la innovación tecnológica, lo que sugiere entonces que la teoría de recursos y capacidades en la que se basa, es el marco teórico principal de la innovación tecnológica. La figura 1 muestra el fundamento teórico de la innovación tecnológica relacionada con los hallazgos encontrados en la revisión de estudios recientes.
En los últimos cien años la escuela neoclásica ha visto al desarrollo de innovaciones tecnológicas como un factor externo al sistema, es decir, un elemento que se desarrolla independientemente de los factores económicos y sociales. Es a través de la teoría neoclásica que se ha tratado de explicar la mayoría de los fenómenos económicos en el mundo. Sin embargo, esta teoría no ha podido explicar la conducta de los factores en el desarrollo tecnológico. Esto es debido a que la teoría neoclásica considera a la innovación como un factor estático, es decir, sin variabilidad, fácil de reproducirse y usarse (Cayama, 2008).

La escuela neoclásica no es la única corriente teórica que ha tratado de explicar el fenómeno de la innovación tecnológica. La escuela evolucionista, a diferencia de la neoclásica, propone empresas diferenciadas tecnológicamente, con habilidades distintas para desarrollar cosas, con diversas capacidades de aprendizaje y

Figura 1. Fundamento teórico de la innovación tecnológica

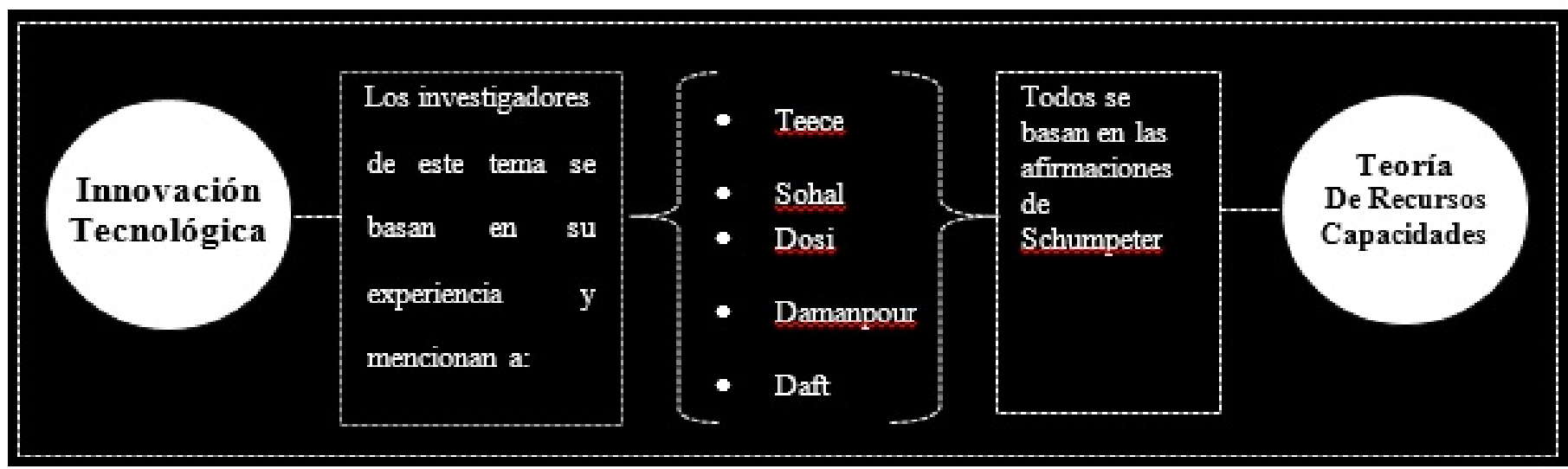

Fuente: elaboración propia

Por otro lado, buscando mayor sustento teórico de la innovación tecnológica, se presenta un análisis de las diferentes bases teóricas en las que se basan los hallazgos de los autores más reconocidos, encontrando las siguientes teorías: neoclásica como principio de la innovación tecnológica; evolucionista, que es como se le llama a la corriente ideológica que siguen los investigadores de la innovación, y de los recursos y capacidades, que es la teoría en que se basan los autores. A continuación se exponen las posturas teóricas encontradas en los estudios.

Teoría neoclásica de la innovación procesamiento de la información. Una de las mayores aportaciones de la escuela evolucionista es la idea de que la innovación no es un elemento fortuito, sino un proceso en el que intervienen varios factores para su desarrollo. El propulsor de esta escuela fue Schumpeter (1934), quien sugiere que la innovación es un factor importante en el desempeño económico de las empresas, ya que él consideraba a la innovación como una pieza clave para el desarrollo del capitalismo y un motor impulsor de la economía.

\section{Teoría evolucionista de la innovación}


Para Schumpeter (1934), la innovación es toda nueva iniciativa capaz de proponer y realizar nuevas combinaciones de medios de producción; por ello, tiene que ver más con una nueva técnica en la producción, promovida de manera intencional, que coloca a la empresa en mejor situación competitiva. Debido a lo anterior, el principal impulsor de la innovación es aquella persona que propone combinaciones en los medios de producción. A este tipo de personas Schumpeter (1934), las denomina empresarios.

Asimismo, las innovaciones más importantes son las radicales, ya que éstas son las que producen cambios revolucionarios; lo anterior se debe a que las nuevas tecnologías sustituyen a las antiguas, a través de un proceso al que llamó destrucción creativa. Otra afirmación de Schumpeter (1934), es que las empresas más grandes tenían ventaja sobre las pequeñas para realizar innovaciones, debido a su poder financiero. Esta afirmación hasta el día de hoy sigue estando en discusión.

Al igual que Schumpeter (1934), Teece (1986) argumenta que las grandes empresas tienen una mayor inversión y activos; debido a ello suelen tener ventaja sobre las pequeñas a la hora de desarrollar innovaciones. Por otro lado, sugiere que la innovación es un proceso que se ve afectado por el aprendizaje, el conocimiento y las habilidades técnicas. Debido a ello, todos los miembros de una empresa son potencialmente innovadores, además de que las empresas desarrollan innovaciones tecnológicas para mantenerse competitivas.

Otra teoría que surge para tratar de complementar la explicación e importancia de la innovación en la empresa es la propuesta de Daft (1978), consistente en una teoría de doble núcleo que propone dos tipos de innovación: la innovación tecnológica y la innovación administrativa. La innovación tecnológica hace referencia a la innovación en productos, servicios y procesos de producción, que son el núcleo de las habilidades técnicas de la organización. La innovación administrativa se refiere a los cambios en la estructura organizacional y procedimientos administrativos. Estas dos formas de innovación varían en importancia, dependiendo de las necesidades de la empresa.

En lo referente a la innovación tecnológica, el desarrollo de este tipo de innovación es un factor preponderante en el desempeño de las empresas, y resulta de una búsqueda de oportunidades de las empresas para mantenerse competitivas, como lo menciona Schumpeter (1934).

Por otra parte, Dosi (1997), sugiere que la innovación tecnológica se desarrolla en base a mecanismos de aprendizaje a partir de una acción productiva o tecnología incorporada. En este caso la innovación se realiza bajo cierta incertidumbre, por lo cual su éxito es difícil de predecir. Debido a lo anterior el departamento de investigación y desarrollo es una pieza fundamental en el desarrollo de innovaciones. Dosi (1997), cuestiona las aseveraciones de la teoría neoclásica y sugiere que el desempeño de la innovación no es predecible.

Damanpour (1991), argumenta la inestabilidad de los diferentes resultados en las investigaciones sobre la innovación esgrimiendo como causa el hecho de que los estudios se realizan desde diferentes perspectivas de investigación, razón por la cual se encuentran diferentes factores determinantes de la innovación en diferentes empresas. Además, comenta que las empresas con una estructura organizacional rígida inhiben las innovaciones en producto.

Otro autor que destaca la relevancia de las ideas de Schumpeter (1934), es Freeman (2003), quien remarca la aparición de numerosos artículos relacionados con que la innovación tecnológica ha llegado a ser un factor crucial para la competitividad y para el crecimiento económico. Y un factor preponderante en el desarrollo de innovaciones tecnológicas es el capital humano calificado.

Para los Autores Nelson y Winter (1991), un factor importante en el desarrollo de innovaciones tecnológicas es el conocimiento; éste es acumulativo a través del tiempo en las empresas. Además, el conocimiento es diferente en cada empresa, aparte de ser una fuente de competitividad. Para estos autores la innovación de las empresas siempre estará rodeada de incertidumbre, y destacan que la inversión en $\mathrm{I}+\mathrm{D}$ siempre será beneficiosa para el desarrollo de innovaciones tecnológicas.

\section{Teoría de recursos y capacidades}

Esta teoría desarrolla conceptos para la búsqueda de la rentabilidad de las empresas. Los elementos que intervienen en el éxito de las empresas son los que corresponden a la organización. Estos elementos deber ser heterogéneos y cumplir con las propiedades de ser valiosos, escasos y difíciles de imitar o de sustituir, (Barney, 1991).

\section{Recursos}

La economía ha determinado como recursos los factores productivos, tierra, capital y trabajo; a nivel estratégico, los recursos productivos de la empresa se clasifican en físicos, financieros, tecnológicos, humanos y de capital. Estos recursos se integran en actividades y rutinas operativas y administrativas denominadas capacidades, (Barney, 1991). 
El término RBV fue acuñado por Wernerfelt (1984), pero Penrose (1959), fue la pionera en el desarrollo de la teoría al establecer: "Una firma es más que una unidad administrativa, es también una colección de recursos productivos dispuestos entre diversos usuarios $\mathrm{y}$ en un cierto plazo, dada una decisión administrativa". Cuando se observa el proceso de negocio de la empresa privada desde este punto de vista, el tamaño de la firma es mejor calibrado a través de la medición de los recursos productivos que emplea.

Weston (1994), sugiere la existencia de diferentes tipos de recursos, como son los físicos; éstos se encuentran compuestos por la tecnología, la planta, el equipo, la localización geográfica, la materia prima, la infraestructura en lotes y las construcciones. En este grupo se encuentran también los recursos financieros, considerados como fuentes de financiamiento de las inversiones de la empresa.

Los recursos de capital humano son las personas que ocupan diferentes cargos dentro de la compañía y sus competencias, entre otros: su grado de capacitación, la experiencia, la toma de decisiones, el potencial de aprendizaje, la capacidad de adaptación, la habilidad de trabajo en equipo, el liderazgo y el espíritu emprendedor, infiere Grant (2006)

Al igual que los recursos intangibles, los recursos humanos son difíciles de valorar. Sin embargo, con la técnica del desarrollo de competencias se ha contribuido a superar este problema. Debido a que con esta técnica se evalúa el grado de cumplimiento de dichos perfiles, se estructuran procesos de capacitación, formación y experiencia que deben cumplirse para lograr que las habilidades técnicas y profesionales del recurso humano se integren a los otros integrantes de la organización para desarrollar las capacidades esperadas y alcanzar los beneficios, indica Grant (2006).

Los recursos de capital organizacional son la estructura organizacional, las líneas de autoridad, la planeación, el control, la coordinación de sistemas, la forma en que los empleados integran sus esfuerzos y las habilidades; no depende sólo de las habilidades de los empleados, sino también del contexto organizacional, propone Grant (2006). La estructura organizacional debe ser flexible, con estructuras horizontales altamente participativas, que motiven la creatividad, la innovación continua y el emprendimiento corporativo, expresa Rialp (2003).

Capacidades

Los recursos aislados no son productivos; las formas en que los recursos interactúan se consideran como capacidades operativas y administrativas; tales recursos están integrados, organizados y se complementan en rutinas organizativas, compuestas por acciones secuenciales, declaran Nelson y Winter (1982). Lo anterior quiere decir que las capacidades se refieren a las habilidades de una empresa para coordinar sus recursos y destinarlos al uso productivo. Estas habilidades se encuentran en las rutinas de una organización, en la forma en que una empresa toma decisiones y maneja sus procesos internos con el fin de lograr objetivos organizacionales. Las capacidades organizativas también se denominan competencias distintivas, y son intangibles y se hallan en la forma de interacción, cooperación y toma de decisiones de los individuos dentro del contexto de una organización.

\section{Autores que se basan en la teoría de recursos y capacidades}

Autores como Daft (1978), Damanpour (1991), Dosi (1997), Sohal et al. (1999) y Teece (1986) también basan sus estudios en la teoría de recursos y capacidades. Cada uno de estos autores ha sido ampliamente citado en estudios relacionados con alguna dimensión o aspecto de la innovación tecnológica.

Teece (1986), enfoca sus estudios en las capacidades tomando como base la teoría de recursos y capacidades. Algunos autores que lo citan como: Un (2010), Pla-Barber et al. (2007), Van de Vrande et al. (2009), Koellinger (2008) y Li et al. (2010), realizan sus estudios tomando el conocimiento como un factor significativo para el desarrollo de innovaciones tecnológicas. En la tabla 1 se desglosan algunos de los autores que citan a Teece (1986), debido a que consideran al conocimiento como una capacidad distintiva que tienen las empresas que también puede ser desarrollada. Además sugieren que las empresas que tienen esta capacidad tienen mayores probabilidades de implementar innovaciones tecnológicas con éxito.

Tabla 1. Autores que citan a Teece.

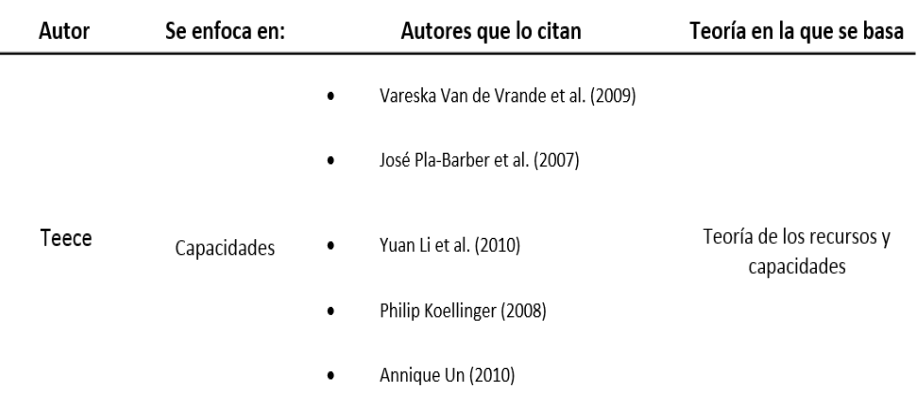


Por su parte, Pekovic et al. (2009) y López et al. (2009), analizan la relación entre las estrategias de calidad y el desarrollo de innovaciones tecnológicas, basados en Sohal et al. (1999), quien indica que las estrategias de calidad pueden inhibir algún tipo de innovación, mientras que otras coadyuvarán al desarrollo de innovaciones. En la tabla 2 se desglosan algunos de los autores que citan a Sohal.

Tabla 2. Autores que citan a Sohal.

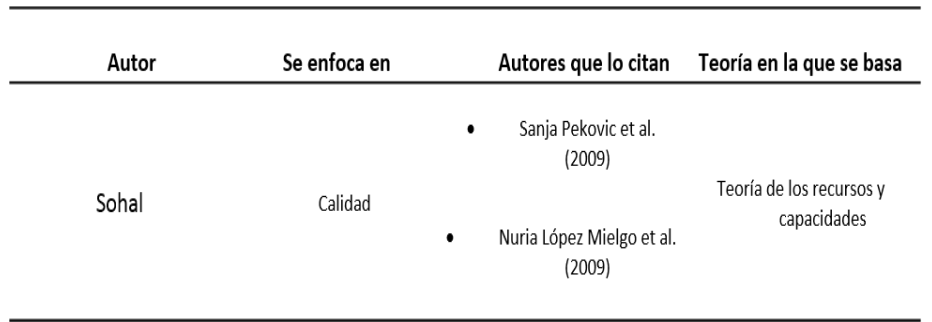

Fuente: Elaboración propia.

Dosi (1997), menciona que el desarrollo de innovaciones tecnológicas se basa en los recursos dedicados para su desarrollo; en estas menciones centran sus investigaciones Vega et al. (2008), Galende et al. (2003) y Shefer et al. (2005), que son acerca de los factores de la empresa para desarrollar innovaciones, de los cuales el principal es el conocimiento. En la tabla 3 se desglosan algunos de los autores que citan a Dosi.

Tabla 3. Autores que citan a Dosi

\begin{tabular}{cccc}
\hline Autor & Se enfoca en & Autores que lo citan & Teoría en la que se basa \\
\hline Dosi & $\begin{array}{c}\text { Cuestiones } \\
\text { organizacionales }\end{array}$ & Jaider Vega Jurado et al. (2008) & \\
& & Jesús Galende et al. (2003) & Teoría de los recursos y \\
capacidades
\end{tabular}

Fuente: Elaboración propia.

De igual manera, Bowen et al. (2009), Gunday et al. (2011) y Jiménez et al. (2011), estudian la relación entre el tipo de organización, el desarrollo de innovaciones y el desempeño de la empresa, teniendo como punto de partida a Damanpour (1991), quien dice que según el tipo de organización y su estructura, ésta desarrollará la innovación que más le convenga. En la tabla 4 se desglosan algunos de los autores que citan a Damanpour.
Tabla 4. Autores que citan a Damanpour.

\begin{tabular}{cccc}
\hline Autor & Se enfoca en & Autores que lo citan & $\begin{array}{c}\text { Teoría en la que se } \\
\text { basa }\end{array}$ \\
\hline Damanpour & $\begin{array}{c}\text { Cuestiones } \\
\text { organizacionales }\end{array}$ & - & Frances E. Bowen et al. (2009) \\
& & Daniel Jiménez Jiménez et al. (2011) & $\begin{array}{c}\text { Teoría de los recursos } \\
\text { y capacidades }\end{array}$ \\
& & Gurhan Gunday et al. (2011) & \\
& &
\end{tabular}

Fuente: Elaboración propia

En cuanto a Daft (1978), sugiere que la estructura organizacional desempeña un rol importante en el desarrollo de innovaciones, Menguc et al. (2010), Wan et al. (2005) y Damanpour et al. (1998), realizan sus análisis sobre el tipo de estructura organizacional y el desarrollo de innovaciones tomando en cuenta las propuestas de Daft (1978). En la tabla 5 se desglosan algunos de los autores que citan a Daft.

Tabla 5. Autores que citan a Daft.

\begin{tabular}{|c|c|c|c|}
\hline Autor & Se enfoca en & Autores que lo citan & $\begin{array}{l}\text { Teoría en la que se } \\
\text { basa }\end{array}$ \\
\hline Daft & $\begin{array}{c}\text { Cuestiones } \\
\text { organizacionales }\end{array}$ & $\begin{array}{ll}\text { - } & \text { Bulent Menguc et al. } \\
& (2010) \\
\text { - } & \text { Fariborz Damanpour et al. } \\
& \text { (1998) } \\
\text { - } & \text { David Wan et al. (2005) }\end{array}$ & $\begin{array}{c}\text { Teoría de los recursos y } \\
\text { capacidades }\end{array}$ \\
\hline
\end{tabular}

Fuente: Elaboración propia.

Otros autores que se basan en las afirmaciones de Schumpeter para realizar sus investigaciones son: Vikash (2010), Stock (2002), Tsai (2005), Laforet (2008) y Tang (2006), que estudian la relación entre el tamaño de la empresa y su relación con el desarrollo de innovaciones. En la tabla 6 se desglosan algunos de los autores que citan a Schumpeter.

Tabla 6. Autores que citan a Schumpeter.

\begin{tabular}{|c|c|c|c|}
\hline Autor & Se enfoca en & Autores que lo citan & Teoría en la que se basa \\
\hline Schumpeter & $\begin{array}{c}\text { Tamaño de la empresa y } \\
\text { capacidades }\end{array}$ & $\begin{array}{l}\text { - } \quad \text { Jianmin Tang (2006) } \\
\text { - } \quad \text { Sylvie Laforet (2008) } \\
\text { - } \quad \text { Kuen-Hung Tsai (2005) } \\
\text { - Gikash Naidoo (2010) } \\
\text { Gregory N. Stock et al. } \\
\text { (2002) }\end{array}$ & $\begin{array}{c}\text { Teoría de los recursos y } \\
\text { capacidades }\end{array}$ \\
\hline
\end{tabular}

Fuente: Elaboración propia. 


\section{Conclusiones}

Desde hace tiempo la innovación se ha visto como una fuente de ventajas competitivas para las empresas. Es Schumpeter (1934) el pionero en las investigaciones y sus beneficios empresariales. Uno de los principales factores que afectan el desarrollo de innovaciones es el tamaño de la empresa argumenta Schumpeter (1934), es decir, que las empresas más grandes tienen mayor facilidad de innovar, al contrario que las empresas pequeñas. Sin embargo, los factores que intervienen para su desarrollo son diversos. A partir de este investigador se han desprendido distintas investigaciones, autores y enfoques teóricos acerca de la innovación.

Uno de los principales enfoques es la Teoría Evolucionista, el cual concibe a la innovación como una cuestión dinámica donde intervienen varios factores para su desarrollo exitoso. Otra teoría es la Neoclásica, es a través de esta teoría de que ha tratado de explicar la mayor parte de los fenómenos económicos mundiales. Sin embargo, esta teoría carece de poder explicativo para la innovación, se puede considerar que esto sucede porque considera a la innovación como un factor estático y sin variabilidad. Por el contrario, la innovación es un fenómeno multivariante debido a la gran cantidad de variables que intervienen para su implementación exitosa, como los son la competencia empresarial, la producción, la investigación y desarrollo (I+D), el conocimiento y la calidad por mencionar algunos.

En la actualidad las empresas tienen una gran necesidad de desarrollar innovaciones para mantenerse competitivas, señala Stock (2002). Esto es debido a que la durabilidad de los procesos y productos es cada vez menor. Por ello, la innovación tecnológica es un proceso fundamental para el desempeño de una empresa. Tal motivo lleva a que las empresas deban seleccionar una estrategia para poder beneficiarse de los resultados de las innovaciones.

Por lo anterior, la estrategia competitiva para desarrollar innovaciones exitosas está relacionada con los recursos y capacidades de la organización. Las organizaciones con un alto grado de desempeño son aquéllas que tienen conductas estratégicas proactivas enfocadas hacia la innovación, sugiere Camison (1997). Asimismo, existe una relación entre la innovación tecnológica y la formulación de los recursos y capacidades.

La innovación es considerada como un determinante de la competitividad empresarial, menciona Barney (1991). Para que una empresa pueda determinar el desempeño es necesaria una estrategia que se enfatice en la tecnología, la administración de recursos humanos y los aspectos organizacionales. Es decir, que desarrolle estrategias para explotar sus recursos y capacidades a fin de que se logre el aprovechamiento de las oportunidades, dice Barney (1991).

De esta manera, la empresa debe utilizar sus recursos y capacidades para mantenerse competitiva y lograr ser mejor que sus competidores. La organización que disponga de una mejor estrategia y realice mejores decisiones para explotar sus recursos y capacidades a fin de desarrollar innovaciones tecnológicas exitosas, será la que tenga mayores probabilidades de mejorar su desempeño y con ello logre una ventaja competitiva sostenible.

\section{Referencias}

Barney, J. 1991. Firm Resources and Sustained Competitive Advantage. Journal of Management, 17, 1, 99-120.

Bowen, F., Rostani, M., Steel, P. 2010. Timing is everything: A metaanalysis of the relationships between organizational performance and innovation. Journal of Business Research, $63,11,1179-1185$.

Camisón, C. 1997. La competitividad de la PYME industrial española: Estrategia y competencias distintivas. Civitas, Madrid.

Cayama, S., Lovera, M., Marín, F. 2008. Innovación tecnológica en la organización empresarial: un análisis desde la teoría biológica evolucionista. Multiciencias, 8, 28-37.

Daft, R. 1978. A Dual- Core Model of Organizational Innovation. Academy of Management Journal, 2, 193-210.

Damanpour, F. 1991. Organizational innovation: A meta-analysis of effects of determinants and moderators. Academy of management journal. 555-590.

Damanpour, F., Gopalakrihnan, S. 1998. Theories of Organizational structure and Innovation Adoption: The Role of Environment Change. Journal of Engineering and Technology Management. 15, 1-24.

Dosi, G. 1997. Perspectives on Evolutionary Theory. Science and Public Policy 18, 353-361.

Freeman, C. 2004, 'Technological infrastructure and international competitiveness', Industrial and Corporate Change. 13, 3, 540-52.

Galende, J., De La Fuente, J. 2003. Internal factors determining a firm's innovative behavior. Research Policy, 32, 715-736.

Gunday, G., Ulusoy, G., Kilic, K., Alpkan, L. 2011. Effects of innovation types on firm performance. Journal Production Economics, 133, 662-676.

Grant, R. 2006. Dirección Estratégica: Conceptos, Técnicas y Aplicaciones. Madrid: Civitas. 
Jimenez, D., Sanz, R. 2011. Innovation, organizational learning, and performance. Journal of Business Research. 64, 408-417.

Koellinger, P. 2008. The relationship between technology, innovation, and firm performance-Empirical evidence from e-business in Europe. Research Policy. 37, 8, 1317-1328.

Li, Y., Su, Z., Liu, Y. 2010. Can Strategic Flexibility help firms profit from product innovation? Technovation. 30, 300-309.

Laforet, S. 2008. Size, strategic, and market orientation affects on innovation. Journal of Business Research. 61, 753- 764 .

López, M., Montes, J., Vázquez, C. 2009. Are Quality and Innovation Management Conflicting Activities? Technovation. 29, 537545.

Menguc, B., Auh, S. 2010. Development and return on execution of product innovation capabilities: The role of organizational structure. Industrial Marketing Management. 39, 820-831.

Nelson, R., Winter, S. 1982. An Evolutinary Theory of Economic Change. Cambridge, M.A: Harvard University Press.

Pla-Barber, J., Alegre, J. 2007. Analysing the link between export intensity, innovation and firm size in a science-based industry. International Business Review. 16, 275- 293.

Pekovic, S., Galia, F. 2009. From quality to innovation: Evidence from two French Employer Surveys. Technovation. 29, 829842

Penrose, E. 1959. The Theory of the Growth of the Firm. John Wiley \& Sons, New York (Edición española: Teoría del Crecimiento de la Empresa, Aguilar, Madrid, 1962).

Rialp, A. 2003. Fundamentos teóricos de la Organización de Empresas. Madrid: Pirámide. 95- 143.

Sohal, A., Gordon, J., Fuller, G., Simon, A. 1999. Manufacturing Practices and Competitive Capability: An Australian Study. Technovation. 19, 295-304.

Teece, D. 1986. Profiting from Technological Innovation: Implications for Integration, Collaboration, Licensing and Public Policy. Research Policy. 15, 6.

Shefer, D., Frenkel, A. 2005. R\&D, firm size and innovation: an empirical analysis. Technovation. 25, 1, 25-32.

Schumpeter, J. 1934. The Theory of Economic Development, Cambridge, MA. Harvard University Press.

Stock, G., Greis, N., Fischer, W. 2002. Firm size and dynamic technological innovation. Technovation. 22, 537-549.

Tang, J. 2006. Competition and innovation behavior. Research Policy.
$35,1,68-82$

Tsai, Kuen-Hung., Wang, Jiann-Chyuan. 2004. The R\&D performance in Taiwan's electronics industry: a longitudinal examination. R\&D Management. 34, 2, 179189.

Un, C., Montoro-Sanchez, M. 2010. Innovative capability development for entrepreneurship: A theoretical framework. Journal of Organizational Change Management. 23, 4, 413-434.

Van de Vrade, V. Vanhaverbeke, W., De Rochemont, M. 2009. Open innovation in SMEs: Trends, motives and management challenges. Technovation. 29, 423-437.

Vega, J., Gutiérrez, A., Fernández de Lucio, I., Manjarrés, L. 2008. The effect of external and internal factors on firms' product innovation. Research Policy. 37, 616- 632.

Vikash, N. 2010. Firm survival through a crisis: The influence of market orientation, marketing innovation and business strategy. Industrial Marketing Management. 39, 1311 1320.

Wan, D., Huat, O., Chin, F. 2005. Determinants of firm innovation in Singapore. Technovation. 25, 261- 268.

Wernerfelt, B. 1980. A resource-based view of the firm. Strategic Management Journal. 5, 2, 171-180.

Weston, J., Brigham, E. 1994. Fundamentos de administración financiera. México: Mc Graw Hill, 1148. 